\title{
Strain Effects in High Temperature Superconductors Investigated With Magneto-Optical Imaging
}

\author{
D. C. van der Laan, H. J. N. van Eck, B. ten Haken, H. H. J. ten Kate, and J. Schwartz, Senior Member, IEEE
}

\begin{abstract}
In order to determine the influence of intermediate deformation steps on the mechanical behavior of Bi-based tapes, the effect of longitudinal applied strain is investigated by means of magneto-optical imaging. The strain is applied in a helium flow-cryostat. Cracks appear soon after the critical current in Bi-based tapes is degraded. All filaments form multiple cracks that grow into tape-wide cracks, running from one filament to the next. The crack location is not caused by stress concentrations in the matrix, but by the mechanically weak colony boundaries. Because of the absence of intermediate rolling steps in the production of $\mathrm{Bi}_{2} \mathrm{Sr}_{2} \mathrm{CaCu}_{2} \mathrm{O}_{x}$ tapes, a different crack structure is observed compared to $\mathrm{Bi}_{2} \mathrm{Sr}_{2} \mathrm{Ca}_{2} \mathrm{Cu}_{3} \mathrm{O}_{x}$ tapes. The relation between the critical current and the formation of cracks is studied. The degradation in critical current before the critical strain is reached may be caused by microcracks that remain undetected by magneto-optical imaging. The influence of strain on the microstructure of $\mathrm{YBa}_{2} \mathrm{Cu}_{3} \mathrm{O}_{x}$ coated conductors is also investigated with magneto-optical imaging. The formation of cracks is believed to be determined by the nickel substrate and related to the Ni-grain size.
\end{abstract}

Index Terms-Crack formation, longitudinal strain, magnetooptical imaging, superconductors.

\section{INTRODUCTION}

A FTER the discovery of high temperature superconductors and the possibility of producing them in long lengths, a large number of power applications come to mind for these ceramic conductors. In some of these applications, such as insert magnets, motors, and generators, the conductor experiences large stresses. In the absence of proper reinforcement, large strain results, and the brittle superconductor will fail. Numerous publications report on the relation of the critical strain $\varepsilon_{c}$ at which the critical current degrades irreversibly, and the pre-compression experienced by the superconductor [1]. In bismuth-based tapes, the pre-compression is induced when the tape is cooled from its reaction temperature to its operation temperature. Experiments show [2] that precompression induces microstructural damage to the conductor.

Earlier work showed [3] that the microstructure in $\mathrm{Bi}_{2} \mathrm{Sr}_{2} \mathrm{Ca}_{2} \mathrm{Cu}_{3} \mathrm{O}_{x}$ (BSCCO 2223) monocore tapes is in-

Manuscript received August 6, 2002. This work was supported in part by the U.S. Air Force Office of Scientific Research and by FOM (Fundamental Research on Matter), which is subsidized by NWO.

D. C. van der Laan is with the University of Twente, Enschede, The Netherlands, and National High Magnetic Field Laboratory, Tallahassee, FL 32310 USA (e-mail: d.c.vanderlaan@tn.utwente.nl).

H. J. N. van Eck, B. ten Haken and H. H. J. ten Kate are with the University of Twente, Enschede, The Netherlands.

J. Schwartz is with the National High Magnetic Field Laboratory, Tallahassee, FL 32310 USA, and also with Department of Mechanical Engineering, FAMU-FSU College of Engineering, Tallahassee, FL 32310 USA.

Digital Object Identifier 10.1109/TASC.2003.812392
TABLE I

SAMPLE PROPERTIES OF THE BSCCO TAPES AND YBCO CC.

\begin{tabular}{lllll}
\hline & Sample 1 & Sample 2 & Sample 3 & Sample 4 \\
\hline Material & $B i-2223$ & $B i-2223$ & $B i-2212$ & YBCO \\
Matrix/Substrate & $A g M g$ & $A g$ & $A g M g$ & $N i$ \\
I $_{\mathrm{c}}$ (sf, 77K) [A] & 30 & 20 & - & 15 \\
$\mathrm{I}_{\mathrm{c}}$ (sf, 4.2K) [A] & - & - & 360 & - \\
Filament number & 19 & 61 & 19 & - \\
\hline
\end{tabular}

fluenced by the deformation steps in the production process. When rolling is used, a large number of microcracks are observed that run transverse to the rolling direction. When the deformation is done by pressing, the microcracks run along the tape length.

Magneto-optical imaging (MOI) [4]-[6] is used to relate the formation of cracks in Bi-based tapes to the production process. Similar studies have been performed [7], [8] where the samples are bent at room temperature, and then inserted into the MOI cryostat. In this work, longitudinal strain is applied to Bi-based tapes within the MOI cryostat while at the same time the crack formation is observed. The relationship between the degradation in $I_{c}$ and the formation of cracks in the superconducting regions was also investigated. The dependence of the critical current on applied strain was investigated using a U-shaped bending spring [9]. A similar study was done on YBCO coated conductors, where it is shown that the formation of cracks is not influenced by the superconducting layer, but dominated by the metallic substrate.

\section{EXPERIMENTAL}

The samples under investigation are BSCCO 2223 tapes with $\mathrm{Ag}$ matrix and AgMg matrix, BSCCO 2212 tapes with $\mathrm{AgMg}$ matrix produced by the powder-in-tube (PIT) method, and YBCO coated conductors (YBCO CC). The YBCO CC consists of buffer layers of $10 \mathrm{~nm} \mathrm{CeO}, 250 \mathrm{~nm} \mathrm{YSZ}$ and $15 \mathrm{~nm} \mathrm{CeO} \mathrm{Ce}_{2}$ deposited on a $50 \mu \mathrm{m}$ nickel substrate that is produced according to the "RABiTS" approach. The $0.3 \mu \mathrm{m}$ YBCO layer is grown on top of the buffer layers using pulsed laser deposition (PLD). Table I gives a description of the properties of the samples, including the critical current at $77 \mathrm{~K}$ in self field. A $1 \mu \mathrm{m}$ thick cap layer of $\mathrm{Ag}$ is deposited on top of the YBCO layer.

Samples of $35 \mathrm{~mm}$ in length are mounted in a helium flow cryostat for straining under the MOI. For YBCO CC, the $10 \mathrm{~mm}$ wide samples are cut along their length in order to fit them on 


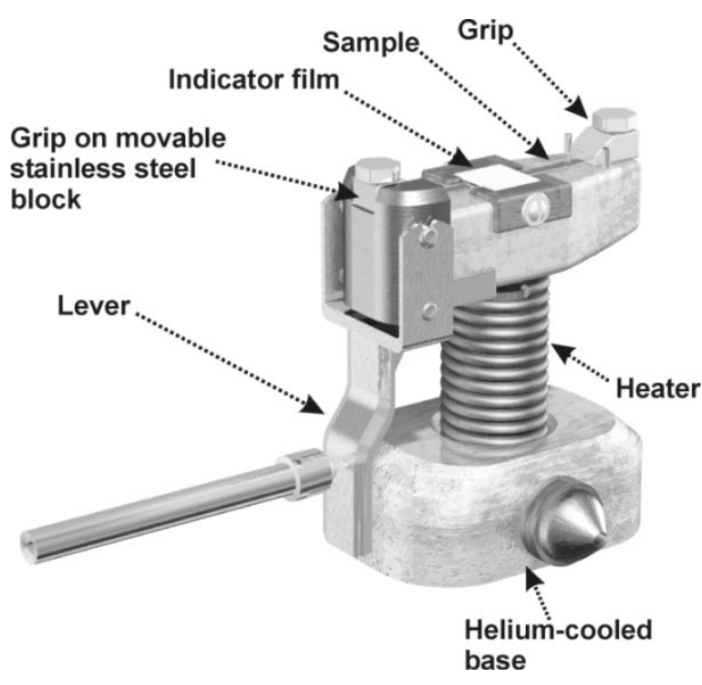

Fig. 1. Sample holder and strain bench located within the helium flowcryostat.

the sample holder. Both ends of the sample are clamped on the holder, using a stationary and a movable grip [10]. Longitudinal strain is applied to the sample by moving one grip using a lever and a stepper motor, as in Fig. 1. The indicator film is located on top of the sample, under the cryostat window. This method enables one to apply strain to the sample while the crack formation is studied at the same time.

After mounting the sample on the strain bench within the MOI cryostat, the sample is cooled to $30 \mathrm{~K}$. Although the sample is mounted with both grips, no additional pre-compression is introduced by the sample holder. One of the grips can move freely during cool-down, and strain is only applied at $30 \mathrm{~K}$ when the stepper motor is activated. After the sample is cooled, magnetic flux is trapped in the superconducting regions of the sample by an external magnetic field of $120 \mathrm{mT}$. These regions are superconducting filaments in the case of BSCCO tapes, and the thin YBCO layer in the case of coated conductors. After removing the magnetic field, the superconducting regions in the sample are visible, even when they are embedded in a silver matrix or under an Ag cap-layer.

Space limitations in the cryostat restrain us from determining the applied strain on the strain bench itself. The applied strain is therefore measured using a strain gage that is glued on the bottom of the sample as indicated in Fig. 2. Because the strain gage reinforces the sample locally, a different type of deformation pattern occur under strain, compared to a bare sample is observed. The reinforcement of a strain gage prevents the tape from failing under the gage. The tape will develop a single crack at the border of the gage due to stress concentrations. To avoid failure outside the indicator film, fiberglass patches are glued on the tape. One set of patches is glued next to the strain gage, on the bottom of the sample, while another set is glued on top of the sample. The patches on top of the sample run from the effective area of the strain gage to the point where they overlap the patches located at the bottom. This configuration ensures the absence of stress concentrations due to the strain gage.

The resolution of the images taken with MOI depends largely on the distance between the indicator film and the trapped flux. When a BSCCO tape is investigated, the superconducting fila-
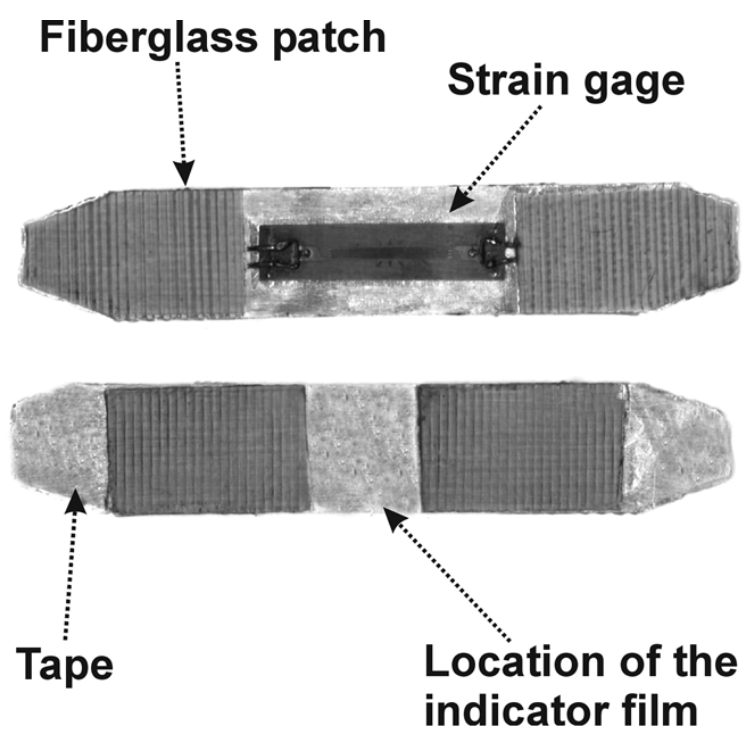

Fig. 2. BSCCO tape with strain gage and fiberglass patches on the bottom and patches on the top, next to where the indicator film will be located.
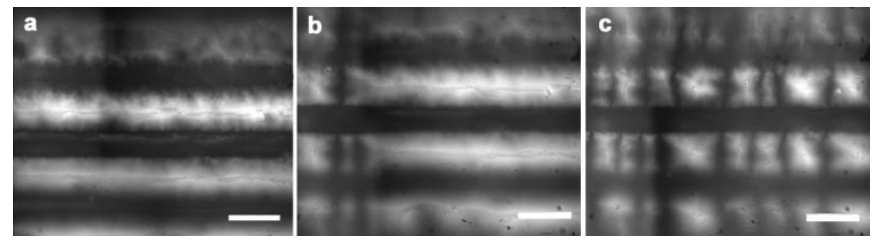

Fig. 3. MO-images of etched sample 1 in remnant field. In the unstrained tape (a), supercurrents are running along the complete length of the filaments, shielding the superconductins from magnetic field. When strain is applied (b), cracks appear in the filaments, and interrupt the current. (c) Additional cracks develop in the filaments, after more strain is applied. Scale bars are $500 \mu \mathrm{m}$ long.

ments are embedded in a $\mathrm{Ag}$ or $\mathrm{AgMg}$ matrix. The minimum distance between the filaments and the indicator film is approximately $20 \mu \mathrm{m}$. To increase the resolution and details of the images, the Ag- or AgMg matrix can be removed using a $\mathrm{NH}_{4} \mathrm{OH} / \mathrm{H}_{2} \mathrm{O}_{2}$ etching solution. Unetched samples are used for determining the crack formation as function of applied strain, although with a poorer resolution.

Lastly, $I_{c}$ as function of strain is measured by soldering the sample on a U-shaped bending spring.

\section{RESULTS AND DISCUSSION}

In this section, the micro-structural damage of the superconductor caused by the applied strain is investigated with MOI. The results are then compared to the degradation in critical current as function of applied strain.

\section{A. Crack Formation in BSCCO Tapes Investigated With MOI}

Fig. 3(a) shows the MO-image of sample 1, after the etched tape is zero-field-cooled (ZFC) to $30 \mathrm{~K}$ and then exposed to a $120 \mathrm{mT}$ field. The trapped magnetic flux in the superconducting filaments is clearly visible in the unstrained tape. Trapped flux appears as bright areas in the image, while the absence of field corresponds to dark areas. The filaments run horizontally in the image. Three complete filaments are visible, while half of a fourth filament can be seen at the bottom of the image. Filaments 


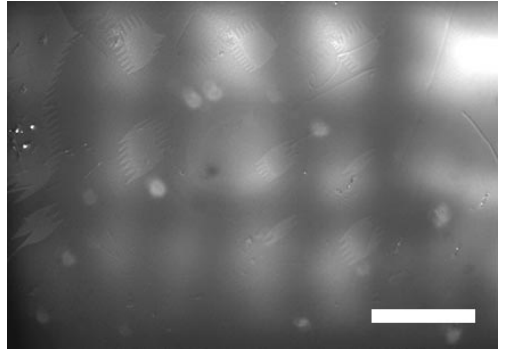

Fig. 4. MO-image of intact sample 1 in remnant field, after a large amount of strain is applied. The scale bar is $500 \mu \mathrm{m}$ long.

from lower layers in the tape are not visible. Detailed structures in the trapped flux are visible, even in the case where no strain is applied to the sample, which indicates that the filaments are not perfectly homogeneous. These structures are an indication for the presence of either microcracks or boundaries between colonies of well-connected grains.

After the sample is strained crack formation in the filaments is observed. This is indicated in Fig. 3(b). Shielding currents that ran over the entire length of the filaments are stopped at cracks. At these locations, trapped flux is released and cracks appear in the image as dark areas. More cracks appear after the strain is increased. Also, filaments that already formed cracks at a lower strain level form new cracks along their length, as can be seen in Fig. 3(c).

A second sample from the same batch with a strain gage and patches is observed next. In this case the silver matrix is left intact to ensure reliable strain data. Fig. 4 shows the resulting MO-image after a large amount of strain is applied. The micro-structural details that were visible in the MO-image of the etched sample are absent in this picture, but the crack pattern is clearly seen and identical to the one of the etched sample. The first three cracks appear at strains of $0.65 \%, 0.73 \%$ and $0.77 \%$.

Although adding a strain gage to, or removing part of the silver sheet of a BSCCO tape influences its mechanical properties, it is still possible to get the same crack pattern from straining an intact sample. If reinforced properly or etched evenly, BSCCO tapes under strain develop fractures within their filaments in a similar manner as a tape in its original state. As soon as the reinforcement is applied incorrectly, or if the sheet of the tape is removed unevenly, the tape breaks at the weakest point due to stress concentrations in the matrix.

The second BSCCO 2223 sample under investigation is sample 2. This sample has a lower critical current than sample 1 and a pure Ag matrix with 61 filaments. The same procedure as with sample 1 is used to investigate the formation of cracks. The levels of applied strain at which the first two cracks appear in the intact sample are $0.61 \%$ and $0.91 \%$. The main difference between the images taken from samples 1 and 2 is the large amount of detail visible within the filaments of sample 2 . The MO-image of a single filament of the sample where the silver matrix is removed is shown in Fig. 5(a). In this case an external magnetic field of $25 \mathrm{mT}$ is applied perpendicular to the tape surface when the image is taken. Well-connected superconducting regions are shielding the external magnetic field and appear as dark areas in the image. The external magnetic field penetrates between the filaments and through

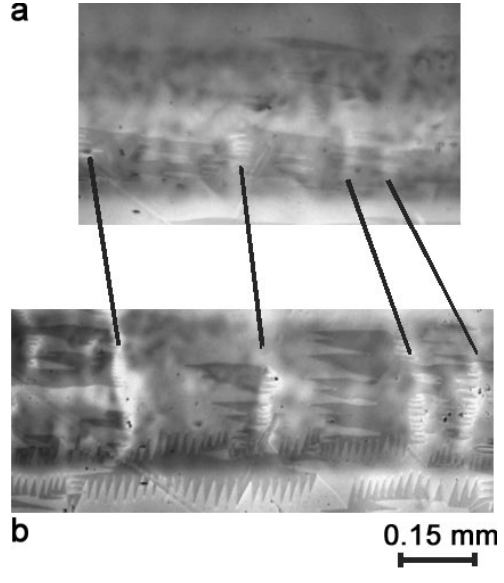

Fig. 5. MO-image of a specific area of sample 2 in an external field of $25 \mathrm{mT}$, (a) before and (b) after strain is applied.

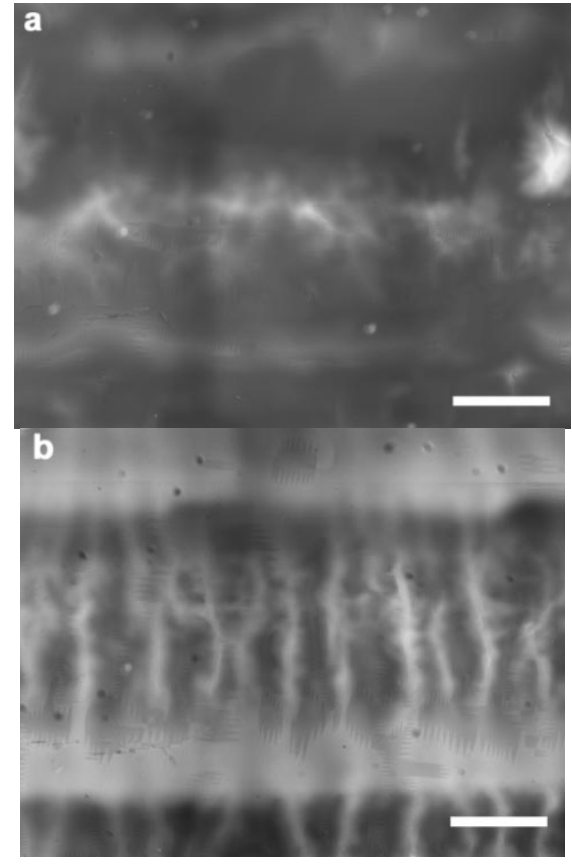

Fig. 6. MO-image of etched sample 3. (a) Before strain is applied in remnant field; (b) In an external magnetic field of $25 \mathrm{mT}$, after strain is applied. The scale bar is $500 \mu \mathrm{m}$.

cracks and colony boundaries within the filaments. These areas appear bright in the image. Colonies of well-connected grains are seen, together with microcracks. It is hard to distinguish between these two forms of micro-structural defects. These defects grow into filament-wide cracks when strain is applied. This is shown in Fig. 5(b), where the same area of the filament in Fig. 5(a) is shown after a large amount of strain is applied.

Fig. 6(a) and (b) show the MO-images of a BSCCO 2212 tape (sample 3) before and after strain is applied. These images are taken when part of the AgMg sheet is removed. The filaments in the tape are difficult to see in these images, since they are very wide $(\sim 0.8 \mathrm{~mm})$ and are partly overlapping each other. The MO-image of the unstrained tape is taken without an external magnetic field present; while a magnetic field of $25 \mathrm{mT}$ is applied perpendicular to the tape surface when the image of the strained tape is taken. The crack formation as a function of 
applied strain is again determined using a strain gage on an intact sample. The values of applied strain at which the first three cracks appear are $0.41 \%, 0.75 \%$ and $0.77 \%$.

\section{B. Relating the Crack Formation to the Production Process}

There is a distinct crack pattern in BSCCO 2223 tapes observed with MOI at strain levels exceeding the critical strain. First of all it is observed that the filaments in the tape show multiple fractures. With increasing strain, more cracks appear in each filament. Although the cracks seem to be distributed randomly at low strain, an average distance between cracks appears at high strain. An average distance between cracks of $200 \mu \mathrm{m}$ is observed for both samples 1 and 2 . The filament width of sample 2 is approximately $200 \mu \mathrm{m}$, resulting in square-shaped filament blocks. The filament width of sample 1 is approximately $450 \mu \mathrm{m}$, but the average distance between cracks is still $200 \mu \mathrm{m}$, indicating that the filament width does not influence on the fracture distance.

Cracks that have formed in one filament migrate to neighboring filaments, growing into tape-wide cracks. The location where the cracks appear in the filaments is related to the boundaries of colonies that are formed between well-connected grains. These boundaries are mechanically weak and therefore most likely to break. Ultrasonic fracturing of filaments in a different study [11] gave the same average colonysize of $200 \mu \mathrm{m}$.

The formation of colony boundaries is influenced by the production process of BSCCO tapes. Intermediate rolling steps are applied to BSCCO 2223 tapes between heat treatments where the 2223 phase is formed from the precursor powder. Not only a better grain alignment is reached through these rolling steps, but also microcracks are formed in the filaments. These cracks are healed partly by the heat treatment that follows the deformation step. These locations will form colony boundaries and are most likely to break. It is possible that the deformation steps have a similar influence on the formation of colony boundaries in neighboring filaments. Filaments will fail at the same location over the width of the tape when strain is applied. The direction of the cracks is normal to the applied strain and the rolling direction.

Due to the absence of intermediate rolling steps in the production process, the colony structure in BSCCO 2212 tapes is different from that in 2223 tapes. The 2212 phase is formed from a liquid, and grain alignment is mediated from the interface with the silver matrix. This results in a much different crack pattern than in 2223 tapes. The cracks are not running perfectly normal to the tape direction but meander through the individual filaments.

\section{Strain Effects in YBCO Coated Conductors}

Applying homogeneous strain to YBCO coated conductors within the MOI setup is more challenging than in the case of BSCCO tapes. First of all, the YBCO samples must be cut in length because their $10 \mathrm{~mm}$ width is too wide for the grips of the strain bench. Second, gluing the fiberglass patches on top of the $1 \mu \mathrm{m}$ thick silver cap layer is problematic. The silver cap layer is peeled off by the patches when strain is applied, resulting in a single crack in the conductor.
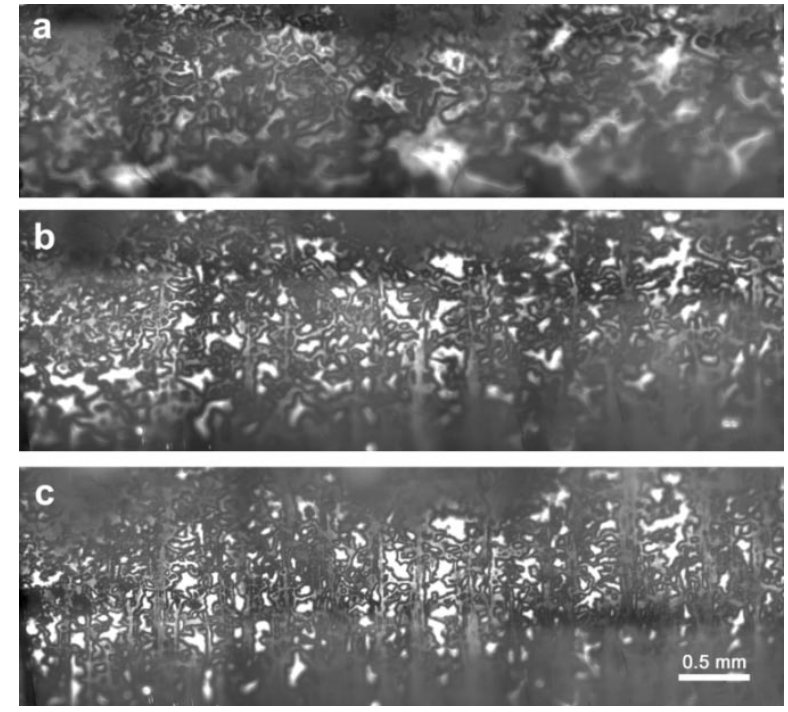

Fig. 7. MO-image of sample 4 in remnant field. (a) The superconducting grains are clearly visible before strain is applied and (b) after a low amount of strain is applied. After a large amount of strain is applied (c), cracks that run through the colonies of well-aligned grains fill the tape.

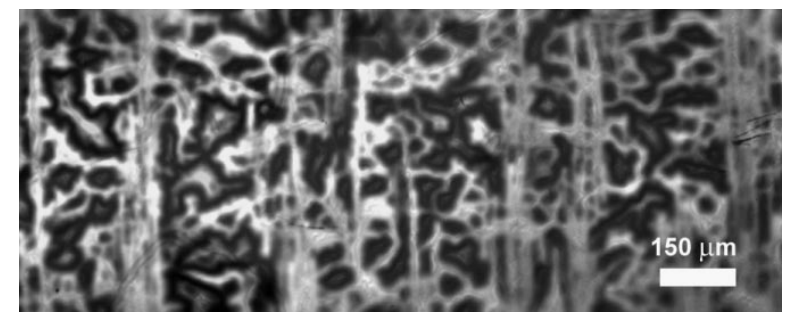

Fig. 8. Smaller area of strained sample YBCO 4. An external magnetic field of $25 \mathrm{mT}$ is applied perpendicular to the tape surface. Flux penetrates through high angle boundaries and cracks.

The thin layer of YBCO that is grown on top of the buffer layers of a coated conductor forms a detailed structure of grains and grain boundaries. Grains, on average several micrometers in size, that are connected at low angle form colonies. These colonies copy the grain structure of the nickel substrate. The colonies themselves are 50-200 $\mu \mathrm{m}$ in size and are partly connected under high angle, determining the quality of the conductor. Previous work [12] showed that colony boundaries that are connected at an angle larger than 2-3 degrees obstruct the supercurrent in passing from one colony to the next.

When using a sample without a strain gage we visualize the crack patterns that develop in the YBCO layer under strain. Fig. 7(a) shows sample 4, before strain is applied. Magnetic flux is trapped in the superconducting YBCO grains. These appear bright, while the grain boundaries appear dark. As seen in Fig. 7(b) and (c), cracks form in the sample after strain is applied. The cracks run normal to the tape direction and the direction of the applied strain. A large number of cracks that cut through superconducting colonies appear in the sample. It appears that the colony structure of the YBCO layer in the tape does not influence the crack locations.

Fig. 8 shows part of the sample shown in Fig. 7(c), when an external magnetic field of $25 \mathrm{mT}$ is applied perpendicular to the tape surface. The colonies of well-connected grains remain shielded from the external magnetic field, while the field 
penetrates the high angle grain boundaries and the cracks in the YBCO layer. This image enables us to determine the distance between cracks to range between $\sim 50 \mu \mathrm{m}$ and $250 \mu \mathrm{m}$.

The cracks are directed perpendicular to the direction of the applied strain, similar to the cracks in BSCCO tapes. The main difference between the crack structure in YBCO CC compared to BSCCO tapes is that the cracks do not only run over colony boundaries, but also run through the colonies themselves. The minimum distance between cracks is around $20-50 \mu \mathrm{m}$, which is the average grain size of the nickel substrate. We therefore conclude that the formation and location of cracks in YBCO coated conductors is not determined by the colony structure in the YBCO layer but by the grain structure in the nickel substrate.

\section{Influence of Applied Strain on $I_{c}$}

Fig. 9(a) and (b) show the normalized critical current as a function of applied strain for both BSCCO 2223 tapes (samples 1 and 2). It is clearly seen that the critical current degrades at low strain values at $77 \mathrm{~K}$, well before the critical strain is reached. A slope of $-7 \%$ in critical current per $\%$ strain is measured for sample 1 , and $-14 \%$ critical current per $\%$ strain for sample 2. Tape 1 with $\mathrm{AgMg}$ matrix shows a critical strain of approximately $0.4 \%$, while tape 2 with a pure silver matrix shows a critical strain of $0.38 \%$. As mentioned earlier, precompression of the filaments determines $\varepsilon_{c}$ for both conductors. In this case the sample holder has the same influence on both tapes, but the matrix material and the filling factor are different. These two factors also influence the pre-compression [13], although the influence of the much larger sample holder dominates.

To compare the crack vs. strain experiment done at $30 \mathrm{~K}$ with MOI and the $I_{c}$ vs. $\varepsilon$ experiment on the brass sample holder, the difference in pre-compression must be determined. Because a strain gage is mounted on the samples for the MOI experiment, the pre-compression of the BSCCO tapes can be determined by measuring the resistance of the strain gage when the sample is cooled from room temperature to $30 \mathrm{~K}$. The change in strain indicated by the strain gage is a combination of the change in properties of the strain gage and the compression of the sample. The thermal output of the strain gage is calibrated by the manufacturer on a 1018 steel sample. The thermal expansion of 1018 steel is well-known, which enables us to calculate the thermal output caused by the change in properties of the strain gage. Knowing this, we can determine the thermal compression of the BSCCO tape.

When cooling sample 1 from room temperature to $30 \mathrm{~K}$, the strain indicated by the strain gage is $-0.29 \%$. We call this the thermal output on BSCCO: $\varepsilon_{M O I}$. The thermal output at $30 \mathrm{~K}$, measured on 1018 steel is $\varepsilon_{\text {Gage }}$, and is $-0.144 \%$. The prestrain of 1018 steel when cooled from $300 \mathrm{~K}$ to $30 \mathrm{~K}$ is $-0.33 \%$. This is determined using the thermal expansion coefficient of 1018 steel. The pre-strain of a tape at $30 \mathrm{~K}$ is thus:

$$
\varepsilon_{\text {Tape }}=\varepsilon_{\text {MOI }}-\left(\varepsilon_{\text {Gage }}-\varepsilon_{\text {Steel }}\right) \text {. }
$$

The pre-compression of the brass bending spring is $-0.65 \%$ at $77 \mathrm{~K}$, and $-0.78 \%$ at $4.2 \mathrm{~K}$, when the sample is soldered on the spring at $450 \mathrm{~K}$. With the assumption that the thermal expansion is a linear function of temperature in the range of $30-450 \mathrm{~K}$, we know the precompression of the bare tape. The
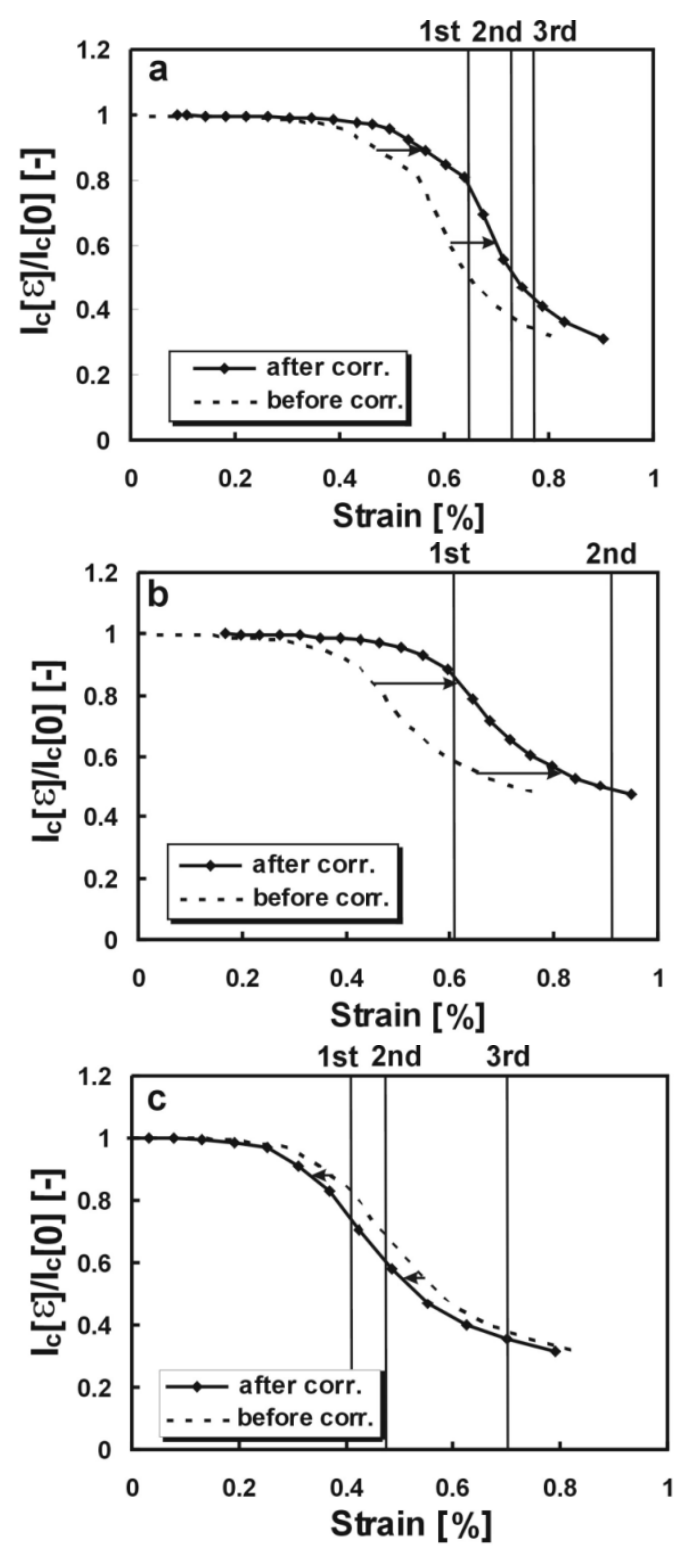

Fig. 9. Critical current vs. strain of sample 1 at $77 \mathrm{~K}$ (a), compared with the results from the MOI experiment at $30 \mathrm{~K}$. The dashed line is the normalized critical current before the difference in pre-compression between both experiments was taken into account. The observed cracks are at an applied strain of $0.65 \%$ (1st), $0.73 \%$ (2nd) and $0.77 \%$ (3rd). Also the results of sample 2 are shown (b); cracks are at $0.61 \%$ (1st) and $0.91 \%$ (2nd). The results for sample 3 are shown (c); cracks are at an applied strain of $0.41 \%$ (1st), $0.475 \%$ (2nd) and $0.7 \%$ (3rd).

resulting difference in pre-compression of samples 1 and 2 is shown by a shift in the $I_{c}-\varepsilon$ curves in Fig. 9. The shift for sample 1 is $+0.1 \%$ and for sample 2 it is $+0.168 \%$.

Similar behavior in critical current as function of applied strain is observed in sample 3. Below the critical strain of $0.25 \%$, a small degradation in $I_{c}$ of $-13 \%$ critical current per $\%$ strain is measured. This is shown in Fig. 9(c), for data at $4.2 \mathrm{~K}$. The difference in pre-compression is taken into account also for this conductor in a similar way as for samples 1 and 2 . This results in a shift of the curve in Fig. 9(c) of $-0.04 \%$.

The microcracks responsible for the small degradation in $I_{c}$ before $\varepsilon_{c}$ is reached cannot be observed with MOI. No change in flux penetration in the filaments is observed at these levels of 


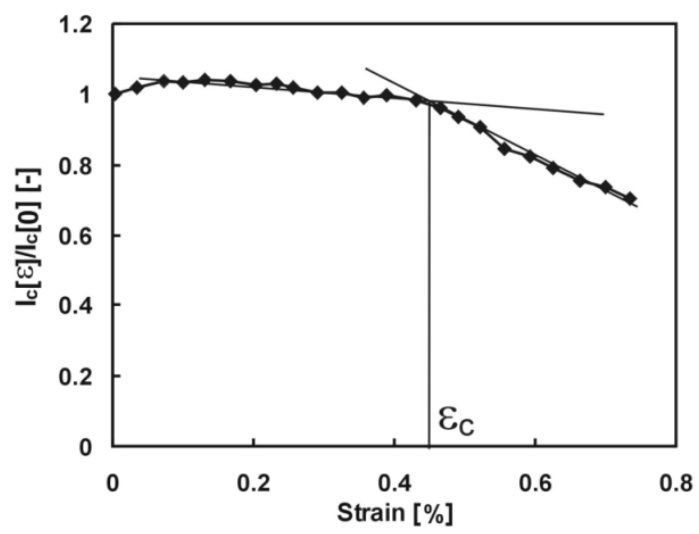

Fig. 10. Degradation in critical current for sample 4.

strain. The thickness and roughness of the filaments keeps the MOI setup from revealing these micro-cracks.

When the formation of cracks observed with MOI is compared to the degradation of the critical current as function of applied strain, some differences are evident. With BSCCO 2212 and 2223 tapes, the first cracks are observed at higher strain levels than the critical strain determined by the bending spring, even after the difference in precompression is taken into account. The critical current of the tapes degraded before any change in flux penetration in the filaments was observed. The critical current of sample 1 degraded $20 \%$ before the first crack was observed. The first crack in sample 2 was observed when the critical current degraded by $15 \%$. For sample 3 , the reduction was $25 \%$. The microcracks responsible for this initial degradation can't be observed by MOI. An alternative explanation is that the first cracks appear in filaments in different filament layers than the top layer. Only the top filament layer is visible with MOI.

The degradation as function of applied strain of sample 4 is shown in Fig. 10, determined on the brass bending spring. The Degradation after the critical strain is reached is less pronounced compared to BSCCO tapes. Since the applied strain at which the first cracks appeared within the MOI could not be measured, we are unable to include these data.

\section{CONCLUSIONS}

The formation of colony boundaries in Bi-based tapes is influenced by the production process of the tape, especially by the intermediate deformation steps applied to BSCCO 2223 tapes. The crack pattern in BSCCO 2212 tapes is different compared to BSCCO 2223 tapes, which is attributed to intermediate rolling steps. Filaments in multifilamentary tapes in both types of BSCCO conducors form multiple cracks when longitudinal strain is applied. The cracks run transverse to the strain direction, and grow into tape-wide cracks when the applied strain is increased. The crack location is determined by the mechanically weak colony boundaries and the presence of microcracks. The average spacing of $200 \mu \mathrm{m}$ between cracks in BSCCO 2223, independent of filament width, agrees with the average colony size found in ultrasonic fracturing experiments.

Cracks only appear in the filaments after the critical current in the tape degraded by $15-25 \%$. Microstructural damage that does not appear in the MO-images may cause the first degradation. It may also be the case that filaments in lower layers which are not visible with MOI form cracks before the filaments in the top layer are damaged.

Although the colony boundaries in BSCCO tapes determine the crack location under strain, in YBCO coated conductor the nickel grain structure dominates the crack formation. This results in a less pronounced degradation in $I_{c}$ when the critical strain is reached. When strain is applied to a coated conductor, cracks that run transverse to the strain direction appear. The cracks run through the colonies of well-connected grains of YBCO. The minimum distance between cracks of $20-50 \mu \mathrm{m}$ correspond to the average nickel grain size.

\section{REFERENCES}

[1] B. ten Haken, A. Beuink, and H. H. J. ten Kate, IEEE Trans. Appl. Supercond., vol. 7, pp. 2034-2037, 1997.

[2] R. Passerini, M. Dhallé, E. Giannini, G. Witz, B. Seeber, and R. Flükiger, Physica C, vol. 371, p. 173, 2002.

[3] J. A. Parrell, A. A. Polyanskii, A. E. Pashitski, and D. C. Larbalestier, Supercond. Sci. and Technol., vol. 9, p. 393, 1996.

[4] M. V. Indenbom, V. I. Nikitenko, A. A. Polyanskii, and V. K. VlaskoVlasov, Cryogenics, vol. 30, p. 747, 1990.

[5] M. V. Indenbom, N. N. Kolesnikov, M. P. Kulakov, I. G. Naumenko, V. I. Nikitenko, A. A. Polyanskii, N. F. Vershinin, and V. K. Vlasko-Vlasov, Physica C, vol. 166, p. 486, 1990.

[6] M. R. Koblischka and R. J. Wijngaarden, Supercond. Sci. and Technol., vol. 8, p. 199, 1995.

[7] M. R. Koblischka, T. H. Johansen, and H. Bratsberg, Supercond. Sci. Technol., vol. 10, p. 693, 1997.

[8] M. Polak, J. A. Parrell, A. A. Polyanskii, A. E. Pashitski, and D. C. Larbalestier, Appl. Phys. Lett., vol. 70, p. 1034, 1997.

[9] B. ten Haken, A. Godeke, and H. H. J. ten Kate, Journal of Appl. Phys., vol. 85, p. 3247, 1999.

[10] D. C. van der Laan, H. J. N. van Eck, M. W. Davidson, B. ten Haken, H. H. J. ten Kate, and J. Schwartz, Physica C, vol. 372, 2002.

[11] D. C. Larbalestier, J. W. A. Anderson, S. E. Babcock, X. Y. Cai, S. E. Dorris, M. Feldmann, J. Jiang, Q. Li, J. A. Parrell, R. Parrella, M. Polak, A. A. Polyanskii, G. N. Riley Jr, M. Rupich, and Y. Wu, Advances in Superconductivity, vol. XI, 1998.

[12] D. T. Verebelyi, D. K. Christen, R. Feenstra, C. Cantoni, A. Goyal, D. F. Lee, M. Paranthaman, P. N. Arendt, R. F. DePaula, J. R. Groves, and C. Prouteau, Appl. Phys. Lett., vol. 76, p. 1755, 2000.

[13] H. Kitaguchi, K. Itoh, T. Takeuchi, K. Togano, and H. Wada, IEEE Trans. on Appl. Supercond., vol. 11, p. 3058, 2001. 